\title{
Inventory Control under Substitutable Demand: A Stochastic Game Application
}

\author{
Zeynep Müge Avṣar, ${ }^{1}$ Melike Baykal-Gürsoy ${ }^{2}$ \\ ${ }^{1}$ Department of Industrial Engineering, Bilkent University, Bilkent, \\ 06533 Ankara, Turkey \\ ${ }^{2}$ Department of Industrial Engineering Rutgers, The State University of New Jersey, \\ Piscataway, New Jersey 08854-8018
}

Received August 1997; revised March 2001; accepted 13 November 2001

\begin{abstract}
Substitutable product inventory problem is analyzed using the concepts of stochastic game theory. It is assumed that there are two substitutable products that are sold by different retailers and the demand for each product is random. Game theoretic nature of this problem is the result of substitution between products. Since retailers compete for the substitutable demand, ordering decision of each retailer depends on the ordering decision of the other retailer. Under the discounted payoff criterion, this problem is formulated as a two-person nonzero-sum stochastic game. In the case of linear ordering cost, it is shown that there exists a Nash equilibrium characterized by a pair of stationary base stock strategies for the infinite horizon problem. This is the unique Nash equilibrium within the class of stationary base stock strategies. (C) 2002 Wiley Periodicals, Inc. Naval Research Logistics 49: 359-375, 2002; Published online in Wiley InterScience (www.interscience.wiley.com). DOI 10.1002/nav.10018
\end{abstract}

Keywords: stochastic games; inventory control; substitutable demand

\section{INTRODUCTION}

This study focuses on investigating the equilibrium strategies for substitutable product inventory control systems within the class of stationary base stock strategies. When different products are sold by different retailers, substitution between these products causes the retailers to decide on their order quantities in a competitive environment, and thus the game theoretic nature of the problem arises. In this article, a nonzero-sum discounted stochastic game formulation is given for the two-product problem. The retailers observe their inventory levels periodically and take actions according to their ordering policies. It is assumed that both retailers behave rationally, i.e., each retailer tries to optimize his own payoff. The setup cost of each retailer to place an order is assumed to be zero.

Substitutable product inventory problem was first studied by McGillivray and Silver [7] in the Economic Order Quantity (EOQ) context. Later, Parlar and Goyal [10] and Khouja, Mehrez, and Rabinowitz [5] gave single-period formulations for an inventory system with two substitutable products independently of each other. In [8], Parlar proposed a Markov Decision Process (MDP)

Correspondence to: Z.M. Avsar

(C) 2002 Wiley Periodicals, Inc. 
model to find the optimal ordering policies for perishable and substitutable products from the point of view of one retailer. Parlar's study in [9] is a game theoretic analysis of the inventory control under substitutable demand. He modeled the two-product single-period problem as a two-person nonzero-sum game and showed that there exists a unique Nash equilibrium. As an extension of the model in [9], Wang and Parlar [15] studied the three-product single-period problem.

In this study, the work in [9] is extended to the infinite horizon and lost sale case. The solution of the corresponding nonzero-sum stochastic game is considered over the class of stationary base stock strategies. This restriction makes both implementation of the strategies and analysis of the problem easier. It is shown that under the discounted payoff criterion retailers possess a stationary base stock Nash strategy pair which is the unique Nash equilibrium within the class of stationary base stock strategies. Stationary base stock strategies are expressed by constant order-upto-levels. If the inventory at the beginning of a period is below the order-upto-level, then an order is given to bring the inventory amount to that level; otherwise, no action is taken. Also, it is observed that cooperation of retailers leads to a better total payoff than the sum of the individual payoff amounts of the retailers in the non-cooperative case.

There are two other models that are related to the substitutable product inventory model. In [14], Veinott considered a single retailer inventory problem under backlogging, budget and/or capacity limitations. He gave conditions to ensure that the base stock ordering policy is optimal for the expected discounted cost criterion. Later, Ignall and Veinott [4] considered the same model and obtained new conditions under which a myopic ordering policy (a policy of minimizing the expected cost in one period only) is optimal for a sequence of periods. An important one of these conditions is the so-called substitute property. This property holds when the myopic policy is such that increasing the initial inventory of any product does not increase the stock on hand after ordering of any other product. This property arises in the models that include some kind of product substitution such as substituting storage space for one product for that of another, or the demand for a product at one location for that at another location in a multi location inventory model. But, the substitution between products in the sense of this paper destroys the optimality of the myopic policy. Since there is only one retailer, there is no competition in this model. In [6], Kirman and Sobel considered a dynamic oligopoly model with inventories. In oligopolies, a small number of firms produce homogeneous or comparable goods competitively. Firms compete by increasing the demand for their product via advertisement, pricing or by keeping inventories. They considered the case of backlogging and discounting, and analyzed this model using the stochastic game approach. For the infinite horizon case, they gave conditions under which the game has a Nash equilibrium and each firm has a base stock type myopic policy. One condition requires that the demand function is smooth. This condition eliminates the cases such as all customers always choose to buy from the firm with the lowest price. Although there is competition in this model, the substitution between products is not considered.

Organization of this article is as follows: The problem and the notation are introduced, and the model is developed in Section 2. Then, in Section 3, analyses are presented for the use of stationary base stock strategies and cooperation of the retailers is discussed.

\section{MODEL OF THE SUBSTITUTABLE PRODUCT INVENTORY PROBLEM}

In the analysis of substitutable product inventory problem over infinite horizon, concepts of nonzero-sum stochastic games are used. Two retailers of different products who compete for the substitutable demand of these products are the players of the game. The mathematical formulation considered is a nonzero-sum game because what is earned (or lost) by one retailer may not be the loss (or earning) of the other retailer although what is earned or lost by each retailer depends on 
both strategies, not the strategy taken by just that retailer. Demand distributions of the products and the substitution rates are known by both players. So, being aware of all of the parameters and the strategies that can be employed by the opponent, each retailer tries to find out the best strategy as a reply to the opponent. Since the retailers somehow agree (although they do take their actions independently in a strictly competitive environment, they know all the parameters that would affect their decisions) on a pair of strategies, called Nash strategies in the context of nonzero-sum games, this pair is said to be an equilibrium point. Unilateral deviations of either of the players from his Nash strategy do not improve his expected payoff. For the nonzero-sum stochastic game formulation in this article, Nash equilibrium is investigated.

Retailers observe their inventory levels at the beginning of each period and make their ordering decisions accordingly. A period is named (indexed) by the number of periods from the beginning of that period until the end of the planning horizon, i.e., period $n$ means that there are $n$ decision epochs to go until the end of the planning horizon. Let $X_{n}$ and $Y_{n}$ be the independently and identically distributed (i.i.d.) random variables denoting the demand for product 1 and 2 , respectively, in period $n$. Product $i$ is sold for $q_{i}$ per unit, $i=1,2$. Ordering cost is a linear function of the order quantity $Q_{i n}$ for product $i, i=1,2$, in period $n$. $c_{i}$, that satisfies $0<c_{i}<q_{i}$, is the ordering cost per unit of product $i, i=1,2$. Orders are delivered instantaneously. $l_{i}$ is the unit lost sale cost, and $h_{i}$ is the inventory holding cost per unit of product $i$ per period. Substitution rates are given as the probabilities that a customer switches from one type of product to the other when the product demanded is sold out. $a(b)$ is the probability that a customer of product 1 (2) switches to product 2 (1) given that product 1 (2) is sold out. Let $I_{n}$ and $J_{n}$ be the inventory levels of retailers I and II, respectively, at the beginning of period $n$. At each epoch $n,\left(I_{n}, J_{n}\right)$ denotes the state of the stochastic process and $\left(Q_{1 n}, Q_{2 n}\right)$ denotes the action pair taken by the retailers. Then, $z_{1 n}=I_{n}+Q_{1 n}$ and $z_{2 n}=J_{n}+Q_{2 n}$ are the inventory levels just after the orders are replenished. The forward dynamic equations associated with the state variables are given as $I_{n-1}=\left[z_{1 n}-x_{n}-b\left[y_{n}-z_{2 n}\right]^{+}\right]^{+}$and $J_{n-1}=\left[z_{2 n}-y_{n}-a\left[x_{n}-z_{1 n}\right]^{+}\right]^{+}$, where $[a]^{+}=\max \{0, a\}$. Note that if retailer II can not satisfy demand $Y_{n}$ fully, then the remaining demand $\left[Y_{n}-z_{2 n}\right]^{+}$switches to product 1 or vice versa. By suppressing subscript $n$, i.e., considering the order-upto-levels as $z_{1}=I+Q_{1}$ and $z_{2}=J+Q_{2}$ when the state is $(I, J)$ and the order quantities are $Q_{1}$ and $Q_{2}$,

$$
\begin{aligned}
P_{(I, J)\left(z_{1}-I, z_{2}-J\right)}^{1}= & c_{1}\left(z_{1}-I\right)+h_{1} E\left(\left[z_{1}-X-b\left[Y-z_{2}\right]^{+}\right]^{+}\right)+l_{1} E\left(\left[X-z_{1}\right]^{+}\right) \\
& -q_{1} E\left(\min \left\{z_{1}, X+b\left[Y-z_{2}\right]^{+}\right\}\right)
\end{aligned}
$$

is the one-period expected payoff for retailer I. For the purpose of understanding dynamics of the problem, three possible cases need to be considered for given demand figures $X=x$ and $Y=y$. First, consider the case $x \leq z_{1}, y \leq z_{2}$. Since demand can be fully satisfied by both retailers, total payoff includes the revenue, holding cost and the ordering cost. In case $x \leq z_{1}, y>z_{2}$, retailer II can satisfy $z_{2}$ customers and lose the remaining $\left(y-z_{2}\right)$. Each unsatisfied customer switches to product 1 with probability $b$. So, the expected demand that switches from product 2 to 1 is $b\left(y-z_{2}\right)$. On the other hand, retailer I satisfies all of the demand that is originally for product 1 and he is left with an inventory equal to $\left(z_{1}-x\right)$. Then, the expected demand $b\left(y-z_{2}\right)$ is fulfilled by retailer $\mathrm{I}$ if the number of units remaining of product 1 , i.e., $\left(z_{1}-x\right)$, is larger than or equal to $b\left(y-z_{2}\right)$. In such a case, retailer I ends up with an inventory of $\left(z_{1}-x\right)-b\left(y-z_{2}\right)$. Otherwise, i.e., if the amount $b\left(y-z_{2}\right)$ demanded is greater than the remaining available amount $\left(z_{1}-x\right)$, then the inventory of the first retailer becomes zero at the beginning of the next period and the unsatisfied demand $b\left(y-z_{2}\right)-\left(z_{1}-x\right)$ is lost. Finally, when $x>z_{1}$, retailer I loses unsatisfied demand of $\left(x-z_{1}\right)$ units. 
For the sake of simplicity of the analysis, assume that the nonnegative random demand variables $X$ and $Y$ have continuous density functions $f$ and $g$, respectively, with finite expectations. Let $f(0)=0, g(0)=0$. The corresponding cumulative and complementary cumulative functions will be denoted by $F, G$ and $\bar{F}, \bar{G}$, respectively. Then, considering the explanation in the previous paragraph, one-period expected payoff can be rewritten as

$$
\begin{aligned}
P_{(I, J)\left(z_{1}-I, z_{2}-J\right)}^{1}= & l_{1} E(X)-\left(q_{1}+l_{1}\right) \int_{0}^{z_{1}} x f(x) d x-\left(q_{1}+l_{1}\right) z_{1} \int_{z_{1}}^{\infty} f(x) d x \\
& +h_{1} \int_{0}^{z_{1}}\left(z_{1}-x\right) f(x) d x+c_{1}\left(z_{1}-I\right) \\
& -\left(q_{1}+h_{1}\right) \int_{0}^{z_{1}} \int_{z_{2}}^{z_{2}+\frac{z_{1}-x}{b}} b\left(y-z_{2}\right) g(y) f(x) d y d x \\
& -\left(q_{1}+h_{1}\right) \int_{0}^{z_{1}} \int_{z_{2}+\frac{z_{1}-x}{b}}^{\infty}\left(z_{1}-x\right) g(y) f(x) d y d x
\end{aligned}
$$

Similarly,

$$
\begin{aligned}
P_{(I, J)\left(z_{1}-I, z_{2}-J\right)}^{2}= & c_{2}\left(z_{2}-J\right)+h_{2} E\left(\left[z_{2}-Y-a\left[X-z_{1}\right]^{+}\right]^{+}\right)+l_{2} E\left(\left[Y-z_{2}\right]^{+}\right) \\
& -q_{2} E\left(\min \left\{z_{2}, Y+a\left[X-z_{1}\right]^{+}\right\}\right)
\end{aligned}
$$

is the second retailer's one-period expected payoff. The model is a nonzero-sum game because the summation $\left(P_{(I, J)\left(z_{1}-I, z_{2}-J\right)}^{1}+P_{(I, J)\left(z_{1}-I, z_{2}-J\right)}^{2}\right)$ is not necessarily equal to zero.

Define $L_{1}\left(z_{1}, z_{2}\right)$ as the immediate (one-period) expected payoff except the ordering cost for retailer I when the order-upto-levels are $z_{1}$ and $z_{2}$, i.e., $P_{(I, J)\left(z_{1}-I, z_{2}-J\right)}^{1}=L_{1}\left(z_{1}, z_{2}\right)+c_{1}\left(z_{1}-\right.$ $I)$. Then, using (1), the following holds:

$$
\begin{aligned}
L_{1}\left(z_{1}, z_{2}\right)= & l_{1} \int_{0}^{z_{1}}\left(z_{1}-x\right) f(x) d x-\left(q_{1}+l_{1}\right) z_{1}+l_{1} E(X) \\
& +\left(q_{1}+h_{1}\right) \int_{0}^{z_{1}} \int_{0}^{z_{2}+\frac{z_{1}-x}{b}}\left(z_{1}-x-b\left[y-z_{2}\right]^{+}\right) g(y) f(x) d y d x .
\end{aligned}
$$

Parlar [9] investigated the substitutable product inventory problem for the single-period case and showed that there exists a unique Nash equilibrium specified with order-upto-levels, say $S_{1}$ and $S_{2}$ for retailers I and II, respectively. For the discrete demand case, it is possible to numerically solve the single-period problem although the size of the state space may make it impractical. Under the long-run average payoff criterion, the nonlinear programming formulation developed by Filar et al. [2] can be used to compute Nash strategies. If the discounted payoff criterion is considered, then NLP due to Raghavan and Filar [11] is available.

\section{STATIONARY BASE STOCK NASH STRATEGIES}

The purpose of this section is to investigate Nash equilibrium of the infinite horizon substitutable product inventory problem within the class of stationary base stock strategies. To this end, first the finite horizon problem is analyzed from the viewpoint of retailer I by assigning a stationary base 
stock strategy to retailer II. The results obtained are then extended for the infinite horizon problem, and it is observed that when retailer II uses a stationary base stock strategy the optimal strategy of retailer I, which would be stationary from standard MDP theory, is also of base stock type. Although stationarity of the response strategy of retailer I is immediate for infinite horizon case as noted above with a reference to the MDP theory, the whole development is to show that this optimal stationary response strategy is observed as a base stock strategy by the convergence of optimal nonstationary order-upto-levels of finite horizon problems. Finally, existence and uniqueness of a Nash solution within the class of stationary base stock strategies are proved.

Let $C_{1 n}\left(I, z_{2}\right)$ represent the minimum expected discounted payoff of retailer I for the remaining $n$ periods until the end of the planning horizon given that the beginning inventory $I_{n}$ of the first retailer is $I$ and the inventory level of product 2 just after the replenishment is $z_{2}$. For player II, $C_{2 n}\left(J, z_{1}\right)$ is defined similarly. The discount factor is assumed stationary and will be denoted by $\gamma, 0<\gamma<1 . C_{1 n}\left(I, z_{2}\right)$ satisfies the following functional equation:

$$
\begin{aligned}
C_{1 n}\left(I, z_{2}\right)=\min _{z_{1} \geq I}\left\{c_{1}\left(z_{1}-I\right)\right. & +L_{1}\left(z_{1}, z_{2}\right) \\
& \left.+\gamma \int_{0}^{\infty} \int_{0}^{\infty} C_{1(n-1)}\left(\left[z_{1}-x-b\left[y-z_{2}\right]^{+}\right]^{+}, z_{2}\right) g(y) f(x) d y d x\right\}
\end{aligned}
$$

where the first two components on the right-hand side correspond to the one-period expected payoff. Here, optimal action of retailer I (the minimizing value of $z_{1}$ in the above equation) is determined for a given order-upto-level $z_{2}$ of retailer II. Relative to the initial inventories of product 2 less than or equal to $z_{2}$, retailer II starts every period with $z_{2}$ units of product 2 . Note that the function that is minimized has a constant part, i.e., $-c_{1} I$, and a variable part, say $D_{1 n}\left(z_{1}, z_{2}\right)$. Thus,

$$
C_{1 n}\left(I, z_{2}\right)=\min _{z_{1} \geq I}\left\{D_{1 n}\left(z_{1}, z_{2}\right)-c_{1} I\right\}
$$

and the minimization is performed only on $D_{1 n}\left(z_{1}, z_{2}\right)$, which is the expected payoff of retailer I for the remaining $n$ periods when the inventory levels are $z_{1}$ and $z_{2}$ at the beginning of period $n$ just after the replenishments. Thus, as in Scarf [12], the results presented in this section are obtained by an inductive analysis of the function

$$
\begin{aligned}
D_{1 n}\left(z_{1}, z_{2}\right)=c_{1} z_{1}+ & L_{1}\left(z_{1}, z_{2}\right) \\
& +\gamma \int_{0}^{\infty} \int_{0}^{\infty} C_{1(n-1)}\left(\left[z_{1}-x-b\left[y-z_{2}\right]^{+}\right]^{+}, z_{2}\right) g(y) f(x) d y d x,
\end{aligned}
$$

for every $n$. If $D_{1 n}\left(z_{1}, z_{2}\right)$ is convex in $z_{1}$ for a given order-upto-level $z_{2}$ of retailer II, then optimal strategy of retailer I is a base stock strategy. Order-upto-level of this strategy is the minimizing point of $D_{1 n}\left(z_{1}, z_{2}\right)$, which will be denoted by $S_{1 n}$. Note that $S_{1 n}$ is a function of $z_{2}$. Lemma 1 shows that for a given $z_{2}$ in $[0, \infty), D_{1 n}\left(z_{1}, z_{2}\right)$ is convex in $z_{1}$ and the minimizing point $S_{1 n}$ is greater than zero. Note that $S_{1 n}>0$ because $\lim _{z_{1} \rightarrow 0} \frac{\partial}{\partial z_{1}} D_{1 n}\left(z_{1}, z_{2}\right)<0$ for every $n$.

LEMMA 1: If retailer II uses a stationary base stock strategy with order-upto-level $z_{2}$, then for $n=1,2, \ldots$

(i) $D_{1 n}\left(z_{1}, z_{2}\right)$ is convex in $z_{1}$,

(ii) $\lim _{z_{1} \rightarrow 0} \frac{\partial}{\partial z_{1}} D_{1 n}\left(z_{1}, z_{2}\right)<0$. 
PROOF: The proof is given by induction on the number of periods remaining, $n$. For $n=$ $1, D_{11}\left(z_{1}, z_{2}\right)=c_{1} z_{1}+L_{1}\left(z_{1}, z_{2}\right)$ holds by taking $C_{10}=0$. The partial derivative of $D_{11}\left(z_{1}, z_{2}\right)$ is given using the Leibnitz's rule of differentiation:

$$
\frac{\partial}{\partial z_{1}} D_{11}\left(z_{1}, z_{2}\right)=c_{1}-l_{1} \int_{z_{1}}^{\infty} f(x) d x-q_{1}+\left(q_{1}+h_{1}\right) \int_{0}^{z_{1}} \int_{0}^{z_{2}+\frac{z_{1}-x}{b}} g(y) f(x) d y d x .
$$

Then, $\lim _{z_{1} \rightarrow 0} \frac{\partial}{\partial z_{1}} D_{11}\left(z_{1}, z_{2}\right)=-\left(q_{1}+l_{1}-c_{1}\right)<0$ because $q_{1}>c_{1}$, and the proof of (ii) is complete. The second partial derivative of $D_{11}\left(z_{1}, z_{2}\right)$ is

$\frac{\partial^{2}}{\partial z_{1}^{2}} D_{11}\left(z_{1}, z_{2}\right)=l_{1} f\left(z_{1}\right)+\left(q_{1}+h_{1}\right)\left(\int_{0}^{z_{1}} g\left(z_{2}+\frac{z_{1}-x}{b}\right) \frac{f(x)}{b} d x+f\left(z_{1}\right) G\left(z_{2}\right)\right) \geq 0$

which proves (i).

Assume that the lemma is true for periods $2,3, \ldots, n$. By the induction assumption, the optimal strategy of retailer I in period $n$ is to order upto $S_{1 n}$ if the inventory level is below $S_{1 n}$ and not to order if it is above $S_{1 n}$. Hence,

$$
C_{1 n}\left(I, z_{2}\right)= \begin{cases}c_{1}\left(S_{1 n}-I\right)+C_{1 n}\left(S_{1 n}, z_{2}\right) & \text { if } I<S_{1 n} \\ -c_{1} I+D_{1 n}\left(I, z_{2}\right) & \text { if } I \geq S_{1 n}\end{cases}
$$

where $c_{1}\left(S_{1 n}-I\right)+C_{1 n}\left(S_{1 n}, z_{2}\right)=-c_{1} I+D_{1 n}\left(S_{1 n}, z_{2}\right)$ for $I<S_{1 n}$.

To show that the lemma is true for period $(n+1), D_{1(n+1)}\left(z_{1}, z_{2}\right)$ is rewritten below using the value of $C_{1 n}$ in (4) by comparing $S_{1 n}$ and the inventory level at the beginning of period $n, I_{n}$. Note that $I_{n}$ may take values below or above $S_{1 n}$. This is because $z_{1}$ may be greater than or equal to $S_{1 n}$ and the demand in period $(n+1)$ determines $I_{n}$. For $z_{1}>S_{1 n}$, the following inequalities are used in writing $D_{1(n+1)}\left(z_{1}, z_{2}\right)$ :

$$
\begin{gathered}
I_{n}=z_{1}-x<S_{1 n} \quad \text { if } z_{1}-S_{1 n}<x \leq z_{1}, \quad 0 \leq y<z_{2}, \\
I_{n}=z_{1}-x \geq S_{1 n} \quad \text { if } 0 \leq x \leq z_{1}-S_{1 n}, \quad 0 \leq y<z_{2}, \\
I_{n}=\left(z_{1}-x\right)-b\left(y-z_{2}\right)<S_{1 n} \quad \text { if } z_{1}-S_{1 n}<x<z_{1}, \quad z_{2} \leq y \leq z_{2}+\frac{z_{1}-x}{b} \\
\text { or } 0 \leq x \leq z_{1}-S_{1 n}, \\
z_{2}+\frac{z_{1}-x-S_{1 n}}{b} \leq y \leq z_{2}+\frac{z_{1}-x}{b}, \\
I_{n}=\left(z_{1}-x\right)-b\left(y-z_{2}\right) \geq S_{1 n} \quad \text { if } 0 \leq x \leq z_{1}-S_{1 n}, \quad z_{2} \leq y \leq z_{2}+\frac{z_{1}-x-S_{1 n} .}{b} .
\end{gathered}
$$

Now, using (4) for each of the ranges of $x$ and $y$ above and then rearranging terms in (3), $D_{1(n+1)}\left(z_{1}, z_{2}\right)$ is written as follows:

$$
D_{1(n+1)}\left(z_{1}, z_{2}\right)=c_{1} z_{1}+L_{1}\left(z_{1}, z_{2}\right)+\gamma D_{1 n}\left(S_{1 n}, z_{2}\right)
$$




$$
\begin{aligned}
& -\gamma c_{1} \int_{0}^{z_{1}} \int_{0}^{z_{2}+\frac{z_{1}-x}{b}}\left(z_{1}-x-b\left[y-z_{2}\right]^{+}\right) g(y) f(x) d y d x \\
& +\gamma \int_{0}^{\left[z_{1}-S_{1 n}\right]^{+}} \int_{0}^{z_{2}+\frac{z_{1}-x-S_{1 n}}{b}}\left(D_{1 n}\left(z_{1}-x-b\left[y-z_{2}\right]^{+}, z_{2}\right)\right. \\
& \left.-D_{1 n}\left(S_{1 n}, z_{2}\right)\right) g(y) f(x) d y d x .
\end{aligned}
$$

Since the last term on the right-hand side of (5) vanishes for $z_{1} \leq S_{1 n}$, consider cases $z_{1}<S_{1 n}$ and $z_{1} \geq S_{1 n}$ separately in the analysis of $D_{1(n+1)}\left(z_{1}, z_{2}\right)$. Let $A_{1}\left(z_{1}, z_{2}\right)$ denote the derivative of $D_{1(n+1)}\left(z_{1}, z_{2}\right)$ with respect to $z_{1}$ for $z_{1}<S_{1 n}$ [the derivative of the first four terms of $D_{1(n+1)}\left(z_{1}, z_{2}\right)$ in (5)], i.e.,

$$
A_{1}\left(z_{1}, z_{2}\right)=c_{1}-l_{1} \int_{z_{1}}^{\infty} f(x) d x-q_{1}+\left(q_{1}+h_{1}-\gamma c_{1}\right) \int_{0}^{z_{1}} \int_{0}^{z_{2}+\frac{z_{1}-x}{b}} g(y) f(x) d y d x .
$$

Then,

$$
\begin{aligned}
& \frac{\partial}{\partial z_{1}} D_{1(n+1)}\left(z_{1}, z_{2}\right)=A_{1}\left(z_{1}, z_{2}\right) \\
& \quad+\gamma \int_{0}^{\left[z_{1}-S_{1 n}\right]^{+}} \int_{0}^{z_{2}+\frac{z_{1}-x-S_{1 n}}{b}} \frac{\partial}{\partial z_{1}} D_{1 n}\left(z_{1}-x-b\left[y-z_{2}\right]^{+}, z_{2}\right) g(y) f(x) d y d x .
\end{aligned}
$$

Note that $A_{1}\left(z_{1}, z_{2}\right)$ is not a function of $n$. Since $S_{1 n}>0$ by the induction assumption, the second term of $\frac{\partial}{\partial z_{1}} D_{1(n+1)}\left(z_{1}, z_{2}\right)$ vanishes at $z_{1}=0$. $D_{1(n+1)}\left(z_{1}, z_{2}\right)$ is decreasing when $z_{1}$ is small because

$$
\lim _{z_{1} \rightarrow 0} \frac{\partial}{\partial z_{1}} D_{1(n+1)}\left(z_{1}, z_{2}\right)=-\left(q_{1}+l_{1}-c_{1}\right)<0,
$$

which means the proof is complete for (ii).

To show (i), the second partial derivative with respect to $z_{1}$ is analyzed. For $z_{1}<S_{1 n}$,

$$
\begin{aligned}
\frac{\partial^{2}}{\partial z_{1}^{2}} D_{1(n+1)} & \left(z_{1}, z_{2}\right)=\frac{\partial}{\partial z_{1}} A_{1}\left(z_{1}, z_{2}\right) \\
& =l_{1} f\left(z_{1}\right)+\left(q_{1}+h_{1}-\gamma c_{1}\right)\left(f\left(z_{1}\right) G\left(z_{2}\right)+\int_{0}^{z_{1}} g\left(z_{2}+\frac{z_{1}-x}{b}\right) \frac{f(x)}{b} d x\right),
\end{aligned}
$$

and it is nonnegative since $q_{1}>c_{1}$ and $\gamma<1$. This means that the function defined by the first four terms of $D_{1(n+1)}\left(z_{1}, z_{2}\right)$ in (5) is convex in $z_{1}$. For $z_{1} \geq S_{1 n}$,

$$
\begin{aligned}
& \frac{\partial^{2}}{\partial z_{1}^{2}} D_{1(n+1)}\left(z_{1}, z_{2}\right) \\
& \quad=l_{1} f\left(z_{1}\right)+\left(q_{1}+h_{1}-\gamma c_{1}\right)\left(f\left(z_{1}\right) G\left(z_{2}\right)+\int_{0}^{z_{1}} g\left(z_{2}+\frac{z_{1}-x}{b}\right) \frac{f(x)}{b} d x\right) \\
& \quad+\gamma\left(G\left(z_{2}\right) f\left(z_{1}-S_{1 n}\right)+\int_{0}^{z_{1}-S_{1 n}} g\left(z_{2}+\frac{z_{1}-x-S_{1 n}}{b}\right) \frac{f(x)}{b} d x\right)
\end{aligned}
$$




$$
\begin{aligned}
& \times\left.\frac{\partial}{\partial z_{1}} D_{1 n}\left(z_{1}, z_{2}\right)\right|_{z_{1}=S_{1 n}} \\
& +\gamma \int_{0}^{z_{1}-S_{1 n}} \int_{0}^{z_{2}+\frac{z_{1}-x-S_{1 n}}{b}} \frac{\partial^{2}}{\partial z_{1}^{2}} D_{1 n}\left(z_{1}-x-b\left[y-z_{2}\right]^{+}, z_{2}\right) g(y) f(x) d y d x,
\end{aligned}
$$

where the last term is nonnegative since, by the induction assumption, $D_{1 n}\left(z_{1}+h, z_{2}\right)$ is convex in $z_{1}$ for any $h$. The first two terms are also nonnegative. $\left.\frac{\partial}{\partial z_{1}} D_{1 n}\left(z_{1}, z_{2}\right)\right|_{z_{1}=S_{1 n}}$ is either zero with a finite $S_{1 n}$ value or negative with infinite $S_{1 n}$. If $S_{1 n}$ is finite, then the third term is zero. Otherwise, the only case that needs to be analyzed is the first case where $z_{1}<S_{1 n}$.

In Lemma 2, it is shown that, for any given nonnegative $z_{2}, D_{1 n}\left(z_{1}, z_{2}\right)$ attains its minimum at a finite $S_{1 n}$. [If $D_{1 n}\left(z_{1}, z_{2}\right)$ has multiple minima, then $S_{1 n}$ is the smallest $z_{1}$ value at which the minimum is attained.] To this end, first behavior of the curve $A_{1}\left(z_{1}, z_{2}\right)=0$ is investigated because the last term of $D_{1(n+1)}\left(z_{1}, z_{2}\right)$ in (6) vanishes for $0 \leq z_{1}<S_{1 n}$ and the first partial derivative of $D_{1(n+1)}\left(z_{1}, z_{2}\right)$ becomes $A_{1}\left(z_{1}, z_{2}\right)$ for every $n \geq 1$. Next, it is proved that the sequence $\left\{S_{1 n}\right\}_{n=1}^{\infty}$ is monotonic nondecreasing and converges to a finite limit as $N \rightarrow \infty$.

LEMMA 2: If retailer II uses a stationary base stock strategy with order-upto-level $z_{2}$, then

(i) $A_{1}\left(z_{1}, z_{2}\right)=0$ at a finite $z_{1}$ value,

(ii) for every $n=1,2, \ldots, D_{1 n}\left(z_{1}, z_{2}\right)$ is minimized at a finite $z_{1}$ value,

(iii) $S_{1(n+1)} \geq S_{1 n}$ for $n=1,2, \ldots$,

(iv) $\left\{S_{1 n}\right\}_{n=1}^{\infty}$ is convergent.

PROOF: (i) First, recall (from the proof of Lemma 1) that the first four terms on the righthand side of (5), of which the first derivative with respect to $z_{1}$ is $A_{1}\left(z_{1}, z_{2}\right)$, is convex in $z_{1}$ for any given $z_{2}$. This convex function is decreasing for very small nonnegative $z_{1}$ values because $\lim _{z_{1} \rightarrow 0} A_{1}\left(z_{1}, z_{2}\right)=-\left(q_{1}+l_{1}-c_{1}\right)<0$. Now, proceed with the analysis of the curve $A_{1}\left(z_{1}, z_{2}\right)=0$ in the $\left(z_{1}, z_{2}\right)$ plane. Implicit differentiation gives the derivative of $z_{2}$ with respect to $z_{1}$ as

$$
\frac{d z_{2}^{1}}{d z_{1}}=\frac{-1}{b}-\frac{l_{1} f\left(z_{1}\right)+\left(q_{1}+h_{1}-\gamma c_{1}\right) f\left(z_{1}\right) G\left(z_{2}\right)}{\left(q_{1}+h_{1}-\gamma c_{1}\right) \int_{0}^{z_{1}} g\left(z_{2}+\frac{z_{1}-x}{b}\right) f(x) d x} .
$$

The superscript 1 is used because it is obtained from the first retailer's cost function. Since $q_{1}>c_{1}$ and $0<\gamma<1, \frac{d z_{2}^{1}}{d z_{1}}$ is negative, which means that the curve $A_{1}\left(z_{1}, z_{2}\right)=0$ is strictly decreasing in the $\left(z_{1}, z_{2}\right)$ plane. Thus, given any $z_{2}$ a lower bound for the $z_{1}$ value, that satisfies $A_{1}\left(z_{1}, z_{2}\right)=0$, is obtained by letting $z_{2}$ go to infinity in $A_{1}\left(z_{1}, z_{2}\right)=0$. Denote this lower bound by $\underline{z}_{1}$. It satisfies $\int_{0}^{\underline{z}_{1}} f(x) d x=\frac{q_{1}+l_{1}-c_{1}}{q_{1}+l_{1}+h_{1}-\gamma c_{1}}$. Similarly, for any given $z_{2}$ let $\bar{z}_{1}$ denote the highest value of $z_{1}$ that satisfies $A_{1}\left(z_{1}, z_{2}\right)=0 . \bar{z}_{1}$ is the solution of $A_{1}\left(z_{1}, 0\right)=0$, i.e.,

$$
\left(q_{1}+h_{1}-\gamma c_{1}\right) \int_{0}^{\bar{z}_{1}} G\left(\frac{\bar{z}_{1}-x}{b}\right) f(x) d x+l_{1} F\left(\bar{z}_{1}\right)=q_{1}+l_{1}-c_{1} .
$$

Since $\frac{q_{1}+l_{1}-c_{1}}{q_{1}+l_{1}+h_{1}-\gamma c_{1}}<1$, it is observed that $\underline{z}_{1}<\infty$. Also, since $\frac{d z_{2}^{1}}{d z_{1}}<0$ at any $\left(z_{1}, z_{2}\right), \bar{z}_{1}<\infty$. Hence, given any $z_{2} \in[0, \infty), A_{1}\left(z_{1}, z_{2}\right)=0$ is satisfied at a finite $z_{11}$ value, say $z_{1 \mid z_{2}}$, between $\underline{z}_{1}$ and $\bar{z}_{1}$. 
(ii) Proof is given by induction. Since

$$
A_{1}\left(z_{1}, z_{2}\right)=\frac{\partial}{\partial z_{1}} D_{11}\left(z_{1}, z_{2}\right)-\gamma c_{1} \int_{0}^{z_{1}} \int_{0}^{z_{2}+\frac{z_{1}-x}{b}} g(y) f(x) d y d x
$$

and $A_{1}\left(z_{1}, z_{2}\right)$ is zero at $z_{1}=z_{1 \mid z_{2}}$,

$$
\left.\frac{\partial}{\partial z_{1}} D_{11}\left(z_{1}, z_{2}\right)\right|_{z_{1}=z_{1 \mid z_{2}}}=\gamma c_{1} \int_{0}^{z_{1 \mid z_{2}}} \int_{0}^{z_{2}+\frac{z_{1 \mid z_{2}}-x}{b}} g(y) f(x) d y d x \geq 0 .
$$

Together with Lemma 1, the relation above shows that $D_{11}\left(z_{1}, z_{2}\right)$ is minimized at a finite $z_{1}$ value, say $S_{11}$, such that $S_{11}<z_{1 \mid z_{2}}$.

By induction, assume that given any $z_{2} \in[0, \infty), D_{1 n}\left(z_{1}, z_{2}\right)$ attains its minimum at a finite value $S_{1 n}$ less than or equal to $z_{1 \mid z_{2}}$, i.e., $\frac{\partial}{\partial z_{1}} D_{1 n}\left(z_{1}, z_{2}\right)=0$ at $z_{1}=S_{1 n}$ such that $S_{1 n} \leq z_{1 \mid z_{2}}$. Note that the integration term in (6) vanishes at $z_{1} \leq S_{1 n}$. If $z_{1}>S_{1 n}$, over the range of $x$ and $y$ values in the double integration term in (6), $\left(z_{1}-x-b\left[y-z_{2}\right]^{+}\right) \geq S_{1 n}$ holds. Since $D_{1 n}\left(z_{1}, z_{2}\right)$ is a convex function of $z_{1}$ and its minimum is achieved at $S_{1 n}, \frac{\partial}{\partial w} D_{1 n}\left(w, z_{2}\right)$ is nonnegative at $w=z_{1}-x-b\left[y-z_{2}\right]^{+}$such that $w \geq S_{1 n}$. Hence, the integration term in (6) is nonnegative for $z_{1}>S_{1 n}$. On the other hand, since $A_{1}\left(z_{1}, z_{2}\right)$ is the first partial derivative of a convex function as pointed out in the proof of Lemma 1 and $A_{1}\left(z_{1 \mid z_{2}}, z_{2}\right)=0, A_{1}\left(z_{1}, z_{2}\right) \geq 0$ for $z_{1} \geq z_{1 \mid z_{2}}$. Then, it is observed that $\frac{\partial}{\partial z_{1}} D_{1(n+1)}\left(z_{1}, z_{2}\right) \geq 0$ for $z_{1} \geq z_{1 \mid z_{2}}$. This shows that $D_{1(n+1)}\left(z_{1}, z_{2}\right)$ is nondecreasing in $z_{1}$ for $z_{1} \geq z_{1 \mid z_{2}}$. By the convexity of $D_{1(n+1)}\left(z_{1}, z_{2}\right)$, the minimizing point of $D_{1(n+1)}\left(z_{1}, z_{2}\right)$, i.e., $S_{1(n+1)}$, is less than or equal to $z_{1 \mid z_{2}}$.

(iii) Since $D_{1(n+1)}\left(z_{1}, z_{2}\right)$ is a convex function and its minimum is achieved at $z_{1}=S_{1(n+1)}$, it is sufficient to show that $\frac{\partial}{\partial z_{1}} D_{1(n+1)}\left(z_{1}, z_{2}\right)<0$ for $0 \leq z_{1}<S_{1 n}, n \geq 1$. When $0 \leq$ $z_{1} \leq S_{1 n}$, the first partial derivative $\frac{\partial}{\partial z_{1}} D_{1(n+1)}\left(z_{1}, z_{2}\right)$ is equal to $A_{1}\left(z_{1}, z_{2}\right)$. Since $A_{1}\left(z_{1}, z_{2}\right)$ is the derivative of a convex function and $S_{1 n} \leq z_{1 \mid z_{2}}$ from (ii), $\frac{\partial}{\partial z_{1}} D_{1(n+1)}\left(z_{1}, z_{2}\right)<0$ for $0 \leq z_{1}<S_{1 n}$. Then, using the convexity of $D_{1(n+1)}\left(z_{1}, z_{2}\right)$ in $z_{1}$, it is clear that $S_{1(n+1)} \geq S_{1 n}$ holds.

(iv) Convergence of $\left\{S_{1 n}\right\}_{n=1}^{\infty}$ results from the observation that $\left\{S_{1 n}\right\}_{n=1}^{\infty}$ is a monotonic nondecreasing sequence in compact space $\left[0, z_{1 \mid z_{2}}\right]$.

Over a finite horizon, say $N$-period horizon, order-upto-levels $S_{1 n}$ for $n=1, \ldots, N$, form optimal nonstationary base stock strategy of retailer I given the second retailer's stationary base stock strategy with order-upto-level $z_{2}$. In order to determine optimal strategy of the first retailer over infinite horizon, the limiting behavior of the payoff function $C_{1 n}\left(I, z_{2}\right)$ is analyzed and the corresponding functional equation is given in Lemma 3.

LEMMA 3: Given the second retailer's stationary base stock strategy with order-upto-level $z_{2}, C_{1 n}\left(I, z_{2}\right)$ converges uniformly for all $I$ in a finite interval. The limit function $C_{1}\left(I, z_{2}\right)$ is a continuous function of $I$ and it is the unique bounded solution to

$$
\begin{aligned}
C_{1}\left(I, z_{2}\right)=\min _{z_{1} \geq I}\left\{c_{1}\left(z_{1}-I\right)\right. & +L_{1}\left(z_{1}, z_{2}\right) \\
& \left.+\gamma \int_{0}^{\infty} \int_{0}^{\infty} C_{1}\left(\left[z_{1}-x-b\left[y-z_{2}\right]^{+}\right]^{+}, z_{2}\right) g(y) f(x) d y d x\right\} .
\end{aligned}
$$


PROOF: In Lemma 2(ii), it is shown that, for any given $z_{2} \in[0, \infty)$, an upper bound for $S_{1 n} \geq 0, n=1,2, \ldots$, is $z_{1 \mid z_{2}}$ which is given by the solution of $A_{1}\left(z_{1}, z_{2}\right)=0$. Also, $\bar{z}_{1}<\infty$ is an upper bound for $z_{1 \mid z_{2}}$. Since the expected values $E(X)$ and $E(Y)$ are also assumed to be finite, $\left|C_{1 n}\left(I, z_{2}\right)\right|$ is bounded for all $I$ in $\left[0, \bar{z}_{1}\right]$.

In order to establish the convergence of $C_{1 n}\left(I, z_{2}\right)$, the notation and the method used by Bellman, Glicksberg, and Gross [1] and later by Iglehart [3] are considered. Let $T_{1}$ be the operator defined as follows:

$$
\begin{aligned}
T_{1}\left(z_{1}, I, C_{1} \mid z_{2}\right)=c_{1}\left(z_{1}-I\right)+ & L_{1}\left(z_{1}, z_{2}\right) \\
& +\gamma \int_{0}^{\infty} \int_{0}^{\infty} C_{1}\left(\left[z_{1}-x-b\left[y-z_{2}\right]^{+}\right]^{+}, z_{2}\right) g(y) f(x) d y d x .
\end{aligned}
$$

By assuming $C_{10}\left(I, z_{2}\right)=0$ for every $I \geq 0$, the optimality equation can be written as $C_{1(n+1)}\left(I, z_{2}\right)=\min _{z_{1} \geq I}\left\{T_{1}\left(z_{1}, I, C_{1 n} \mid z_{2}\right)\right\}$ for every $n$. Let $z_{1 n}^{I}$ denote the optimal $z_{1}$ value given the initial inventory is $I$. Note that, from Lemmas 1 and 2 ,

$$
z_{1 n}^{I}= \begin{cases}S_{1 n} & \text { if } I<S_{1 n} \\ I & \text { if } I \geq S_{1 n}\end{cases}
$$

By the optimality of $z_{1(n+1)}^{I}$ and $z_{1 n}^{I}$ in periods $(n+1)$ and $n$, respectively,

$$
\begin{gathered}
C_{1(n+1)}\left(I, z_{2}\right)=T_{1}\left(z_{1(n+1)}^{I}, I, C_{1 n} \mid z_{2}\right) \leq T_{1}\left(z_{1 n}^{I}, I, C_{1 n} \mid z_{2}\right), \\
C_{1 n}\left(I, z_{2}\right)=T_{1}\left(z_{1 n}^{I}, I, C_{1(n-1)} \mid z_{2}\right) \leq T_{1}\left(z_{1(n+1)}^{I}, I, C_{1(n-1)} \mid z_{2}\right)
\end{gathered}
$$

hold. These relations above imply

$$
\left|C_{1(n+1)}\left(I, z_{2}\right)-C_{1 n}\left(I, z_{2}\right)\right| \leq \max _{k=n, n+1}\left\{\left|T_{1}\left(z_{1 k}^{I}, I, C_{1 n} \mid z_{2}\right)-T_{1}\left(z_{1 k}^{I}, I, C_{1(n-1)} \mid z_{2}\right)\right|\right\} .
$$

Here, even a looser upper bound for $\left|C_{1(n+1)}\left(I, z_{2}\right)-C_{1 n}\left(I, z_{2}\right)\right|$ is observed as

$$
\begin{aligned}
\max _{k=n, n+1}\left\{\gamma \int_{0}^{\infty} \int_{0}^{\infty} \mid C_{1 n}\left(\left[z_{1 k}^{I}-x-b\left[y-z_{2}\right]^{+}\right]^{+}, z_{2}\right)\right. \\
\left.\quad-C_{1(n-1)}\left(\left[z_{1 k}^{I}-x-b\left[y-z_{2}\right]^{+}\right]^{+}, z_{2}\right) \mid g(y) f(x) d y d x\right\} .
\end{aligned}
$$

Then,

$$
\begin{aligned}
\max _{0 \leq I \leq \bar{z}_{1}}\left\{\mid C_{1(n+1)}\left(I, z_{2}\right)\right. & \left.-C_{1 n}\left(I, z_{2}\right) \mid\right\} \\
& \leq \gamma \max _{0 \leq I \leq \bar{z}_{1}}\left\{\left|C_{1 n}\left(I, z_{2}\right)-C_{1(n-1)}\left(I, z_{2}\right)\right|\right\} \leq \gamma^{n} \max _{0 \leq I \leq \bar{z}_{1}}\left\{\left|C_{11}\left(I, z_{2}\right)\right|\right\}
\end{aligned}
$$

for $n=1,2, \ldots$, where the second inequality is obtained by using the first one successively. Since $\left|C_{11}\left(I, z_{2}\right)\right|$ is bounded for all $I$ in $0 \leq I \leq \bar{z}_{1}$, the series $\sum_{n=0}^{\infty}\left|C_{1(n+1)}\left(I, z_{2}\right)-C_{1 n}\left(I, z_{2}\right)\right|$ converges for $0 \leq I \leq \bar{z}_{1}$, which means that the series $\sum_{n=0}^{\infty}\left(C_{1(n+1)}\left(I, z_{2}\right)-C_{1 n}\left(I, z_{2}\right)\right)$ converges absolutely. This implies that $\lim _{n \rightarrow \infty}\left(C_{1(n+1)}\left(I, z_{2}\right)-C_{1 n}\left(I, z_{2}\right)\right)=0$. As a result, $C_{1 n}\left(I, z_{2}\right)$ converges for every $I$ in the finite interval $\left[0, \bar{z}_{1}\right]$. Note that the convergence is uniform. 
From (4), one can easily observe that $C_{1 n}\left(I, z_{2}\right)$ is a continuous function. This leads to the continuity of the limit function $C_{1}\left(I, z_{2}\right)$.

In order to show that $C_{1}\left(I, z_{2}\right)$ satisfies the functional equation given in the lemma, consider

$$
\lim _{n \rightarrow \infty} \min _{z_{1} \geq I}\left\{T_{1}\left(z_{1}, I, C_{1(n-1)} \mid z_{2}\right)\right\}=\lim _{n \rightarrow \infty} C_{1 n}\left(I, z_{2}\right) .
$$

Here, for any finite $I$, the minimization operation above would be over a finite interval of $z_{1}$ values. Note that $z_{1 n}^{I} \leq \max \left\{I, \bar{z}_{1}\right\}$ regardless of the value of $z_{2}$. Also, $T_{1}\left(z_{1}, I, C_{1(n-1)} \mid z_{2}\right)$ is a continuous function of $z_{1}$ for any given $I$. Hence, the limit and the minimization operations can be interchanged as follows:

$$
\min _{z_{1} \geq I}\left\{\lim _{n \rightarrow \infty} T_{1}\left(z_{1}, I, C_{1(n-1)} \mid z_{2}\right)\right\}=C_{1}\left(I, z_{2}\right) .
$$

For $I$ being restricted to the interval $\left[0, \bar{z}_{1}\right]$, by the bounded convergence theorem, the limit operation and the double integral in $T_{1}\left(z_{1}, I, C_{1(n-1)} \mid z_{2}\right)$ can be interchanged and the above relation can be written as

$$
C_{1}\left(I, z_{2}\right)=\min _{z_{1} \geq I}\left\{T_{1}\left(z_{1}, I, \lim _{n \rightarrow \infty} C_{1(n-1)} \mid z_{2}\right)\right\}=\min _{z_{1} \geq I}\left\{T_{1}\left(z_{1}, I, C_{1} \mid z_{2}\right)\right\} .
$$

Since $C_{1 n}\left(I, z_{2}\right)$ is a contraction mapping, by the fixed point theorem $C_{1}\left(I, z_{2}\right)$ is the unique bounded solution.

The next step is to determine optimal strategy of retailer I for infinite horizon problem as a response to his opponent's stationary base stock strategy with order-upto-level $z_{2}$. For this purpose, behavior of $\lim _{n \rightarrow \infty} D_{1 n}\left(z_{1}, z_{2}\right)$, to be denoted as $D_{1}\left(z_{1}, z_{2}\right)$, is investigated and it is observed that this limiting function is minimized at $\lim _{n \rightarrow \infty} S_{1 n}$.

LEMMA 4: Over infinite horizon, if retailer II uses a stationary base stock strategy with orderupto-level $z_{2}$, then the first retailer's optimal strategy is also a stationary base stock strategy with order-up-to-level $z_{1 \mid z_{2}}$.

PROOF: The proof is based on the analysis of $\lim _{n \rightarrow \infty} D_{1 n}\left(z_{1}, z_{2}\right)$. Using the bounded convergence theorem for the double integration term of $\underset{D_{1 n}}{D_{n}}$, one obtains

$D_{1}\left(z_{1}, z_{2}\right)=c_{1} z_{1}+L_{1}\left(z_{1}, z_{2}\right)+\gamma \int_{0}^{\infty} \int_{0}^{\infty} C_{1}\left(\left[z_{1}-x-b\left[y-z_{2}\right]^{+}\right]^{+}, z_{2}\right) g(y) f(x) d y d x$.

$D_{1}\left(z_{1}, z_{2}\right)$ is convex because it is the limit of a sequence of convex functions.

From Lemma 2, $\left\{S_{1 n}\right\}_{n=1}^{\infty}$ is a monotonic nondecreasing sequence. In order to show that $\lim _{n \rightarrow \infty} S_{1 n}$ is $z_{1 \mid z_{2}}$, one needs to prove that $z_{1 \mid z_{2}}$ is the least upper bound for the range of sequence $\left\{S_{1 n}\right\}_{n=1}^{\infty}$.

Suppose $z \in\left(0, z_{1 \mid z_{2}}\right)$ is the least upper bound for the range of $\left\{S_{1 n}\right\}_{n=1}^{\infty}$. Then, using (4) to write $C_{1}\left(I, z_{2}\right)$ in terms of $D_{1}\left(I, z_{2}\right)$ when $I$ is compared with $z$,

$$
\begin{aligned}
\frac{\partial}{\partial z_{1}} D_{1}\left(z_{1}, z_{2}\right)= & A_{1}\left(z_{1}, z_{2}\right) \\
& +\gamma \int_{0}^{\left[z_{1}-z\right]^{+}} \int_{0}^{z_{2}+\frac{z_{1}-x-z}{b}} \frac{\partial}{\partial z_{1}} D_{1}\left(z_{1}-x-b\left[y-z_{2}\right]^{+}, z_{2}\right) g(y) f(x) d y d x
\end{aligned}
$$


is obtained. By Lemma 2(iii), if $z$ is used as the order-upto-level in a period, the optimal orderupto-level for the next period would be greater than $z$. Thus, a point in $\left(0, z_{1 \mid z_{2}}\right)$ can not be the least upper bound. Since $S_{1 n} \leq z_{1 \mid z_{2}}$ for every $n, z_{1 \mid z_{2}}$ is the least upper bound.

In order to show that $z_{1 \mid z_{2}}$ is the solution for the infinite horizon problem, replace $z$ with $z_{1 \mid z_{2}}$ in the derivative equation above. For $0 \leq z_{1}<z_{1 \mid z_{2}}, A_{1}\left(z_{1}, z_{2}\right)$ takes negative values. At $z_{1}=z_{1 \mid z_{2}}$, the first derivative of $D_{1}\left(z_{1}, z_{2}\right)$ with respect to $z_{1}$ is $A_{1}\left(z_{1 \mid z_{2}}, z_{2}\right)$ which is zero. Then, since $D_{1}\left(z_{1}, z_{2}\right)$ is convex, $z_{1 \mid z_{2}}$ is the smallest minimizing point.

Lemma 4 implies that if one retailer restricts himself to stationary base stock strategies and if this is declared by that retailer, his opponent can also restrict himself to the stationary base stock strategies for optimizing his payoff.

The results given above are all obtained under the assumption that retailer II uses a stationary base stock strategy. If the first retailer's strategy is given as a stationary base stock strategy, then the same results follow for retailer II. Based on these observations, in the remaining of this section it is shown that there exists a Nash equilibrium which is unique within the class of stationary base stock strategies. Below, Nash equilibrium of stationary base stock strategies is defined for the two-person nonzero-sum stochastic game formulation of the infinite horizon substitutable product inventory control problem. The payoff functions of this game are $D_{1}$ and $D_{2}$, the latter of which is given by the limit of $D_{2 n}$ as $n$ tends to infinity.

DEFINITION 1: $\left(S_{1}^{*}, S_{2}^{*}\right)$ is called a Nash equilibrium relative to initial inventory levels $\left[0, S_{1}^{*}\right] \times\left[0, S_{2}^{*}\right]$ if $D_{1}\left(S_{1}^{*}, S_{2}^{*}\right) \leq D_{1}\left(z_{1}, S_{2}^{*}\right)$, for all $z_{1} \geq 0$, and $D_{2}\left(S_{1}^{*}, S_{2}^{*}\right) \leq D_{2}\left(S_{1}^{*}, z_{2}\right)$, for all $z_{2} \geq 0$.

Nash condition implies that if a retailer takes his Nash strategy, his opponent cannot improve his payoff by taking any strategy other than his Nash strategy.

Before proceeding with the main theorem regarding the existence and uniqueness of a Nash equilibrium of the substitutable product inventory problem within the class of stationary base stock strategies, it should be pointed out that, from now on, the case with infinite order quantities will not be considered.

REMARK 1: If one of the retailers gives an order of infinite units, then he can satisfy every customer for his product, i.e., no one switches to the other product. In such a case, the other retailer would not have any hope of having substitutable demand and so he decides to satisfy only the demand for his product. In other words, for this retailer the problem reduces to a single player problem. The former retailer goes into bankruptcy because the expected value of demand is finite for each product. Hence, if a retailer orders infinitely many units, then his cost becomes infinite (and this is the worst he could do).

This remark leads to another way of observing the validity of Lemma 2(ii) for the case a retailer orders infinite units. Then, this retailer's cost becomes infinity regardless of his opponent's orderupto-level, i.e., $\lim _{z_{1} \rightarrow \infty} D_{1 n}\left(z_{1}, z_{2}\right)=\infty$ for all $z_{2} \geq 0$ and $\lim _{z_{2} \rightarrow \infty} D_{2 n}\left(z_{1}, z_{2}\right)=\infty$ for all $z_{1} \geq 0$.

As shown before (in the proof of Lemma 2), for any order-upto-level $z_{2} \in[0, \infty)$ of the second retailer, retailer I chooses his own order-upto-level in the finite interval $\left[0, \bar{z}_{1}\right]$. Such bounds are obtained also for the second retailer. For a given $z_{1} \in[0, \infty)$, let

$$
A_{2}\left(z_{1}, z_{2}\right)=c_{2}-l_{2} \int_{z_{2}}^{\infty} g(y) d y-q_{2}+\left(q_{2}+h_{2}-\gamma c_{2}\right) \int_{0}^{z_{2}} \int_{0}^{z_{1}+\frac{z_{2}-y}{a}} f(x) g(y) d x d y .
$$


Then, the implicit differentiation of $A_{2}\left(z_{1}, z_{2}\right)=0$ gives

$$
\frac{d z_{2}^{2}}{d z_{1}}=\frac{-\left(q_{2}+h_{2}-\gamma c_{2}\right) \int_{0}^{z_{2}} f\left(z_{1}+\frac{z_{2}-y}{a}\right) g(y) d y}{\left(q_{2}+h_{2}-\gamma c_{2}\right)\left(g\left(z_{2}\right) F\left(z_{1}\right)+\int_{0}^{z_{2}} f\left(z_{1}+\frac{z_{2}-y}{a}\right) \frac{g(y)}{a} d y\right)+l_{2} g\left(z_{2}\right)} .
$$

By symmetry, $A_{2}\left(z_{1}, z_{2}\right)=0$ is also a strictly decreasing curve in the $\left(z_{1}, z_{2}\right)$ plane. This can be seen by observing the validity of the discussion in the proof of Lemma 2 when $z_{1}$ is fixed in $D_{2(n+1)}\left(z_{1}, z_{2}\right)$. The lower bound $\underline{z}_{2}$ for $z_{2 \mid z_{1}}$ is given by the solution of $\lim _{z_{1} \rightarrow \infty} A_{2}\left(z_{1}, z_{2}\right)=0$. Then, $\int_{0}^{\underline{z}_{2}} g(y) d y=\frac{q_{2}+l_{2}-c_{2}}{q_{2}+l_{2}+h_{2}-\gamma c_{2}}<1$ and so $\underline{z}_{2}$ is finite. Similarly, the upper bound $\bar{z}_{2}$ is obtained when $z_{1}=0$ in $A_{2}\left(z_{1}, z_{2}\right)=0$. Also, $\bar{z}_{1}$ is finite because $\frac{d z_{2}^{2}}{d z_{1}}<0$ and $\underline{z}_{1}$ is finite.

Nash strategies of the retailers within the class of stationary base stock strategies are characterized in Theorem 1.

THEOREM 1: The infinite horizon substitutable product inventory control problem has a Nash equilibrium characterized by stationary order-upto-levels, say $S_{1}^{*}$ and $S_{2}^{*}$, relative to the initial inventory levels $I \leq S_{1}^{*}$ and $J \leq S_{2}^{*}$ of retailers I and II, respectively. This is the unique Nash equilibrium within the class of stationary base stock strategies.

PROOF: Suppose that $\left(S_{1}^{*}, S_{2}^{*}\right)$ is a solution of $A_{1}\left(z_{1}, z_{2}\right)=0$ and $A_{2}\left(z_{1}, z_{2}\right)=0$ for

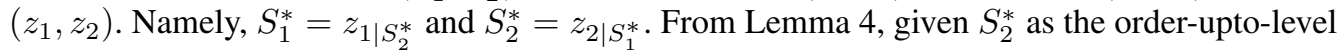
of the second retailer's stationary base stock strategy, $D_{1}\left(z_{1}, S_{2}^{*}\right)$ is a convex function that is

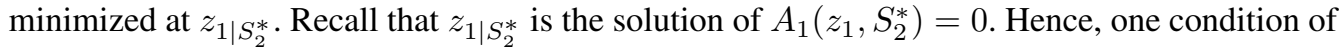

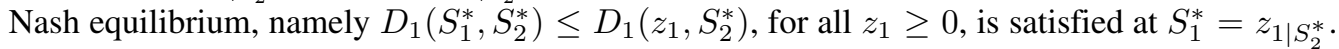
Similarly, given $S_{1}^{*}$ as the first retailer's order-upto-level, $D_{2}\left(S_{1}^{*}, z_{2}\right)$ is convex and its minimizing point $z_{2 \mid S_{1}^{*}}$ is obtained by solving $A_{2}\left(S_{1}^{*}, z_{2}\right)=0$. Thus, the other Nash condition also holds,

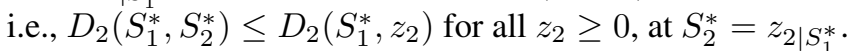

The next step is to consider the existence of a pair $\left(S_{1}^{*}, S_{2}^{*}\right)$ that satisfies both $A_{1}\left(S_{1}^{*}, S_{2}^{*}\right)=0$ and $A_{2}\left(S_{1}^{*}, S_{2}^{*}\right)=0$. Recall from the proof of Lemma 2(i) that the curve $A_{1}\left(z_{1}, z_{2}\right)=0$ is strictly decreasing, and for every $z_{2}$ in $[0, \infty), z_{1 \mid z_{2}}$ takes values between $\underline{z}_{1}$ and $\bar{z}_{1}<\infty$. Similarly, given any $z_{1} \in[0, \infty)$, the analysis of $A_{2}\left(z_{1}, z_{2}\right)=0$ gives the lower and upper bounds $\underline{z}_{2}$ and $\bar{z}_{2}<\infty$, respectively, for $z_{2 \mid z_{1}}$. To prove the existence and uniqueness of the Nash solution one needs to show that there exists only one point, $\left(S_{1}^{*}, S_{2}^{*}\right)$, at which both $A_{1}\left(S_{1}^{*}, S_{2}^{*}\right)=0$ and $A_{2}\left(S_{1}^{*}, S_{2}^{*}\right)=0$ hold. This is true only if the curve $A_{1}\left(z_{1}, z_{2}\right)=0$ is decreasing faster than $A_{2}\left(z_{1}, z_{2}\right)=0$ in the $\left(z_{1}, z_{2}\right)$ plane. Compare $\frac{d z_{2}^{1}}{d z_{1}}$ and $\frac{d z_{2}^{2}}{d z_{1}}$ using the method in [9]. Let

$$
\begin{gathered}
K=\left(q_{1}+h_{1}-\gamma c_{1}\right) f\left(z_{1}\right) G\left(z_{2}\right)>0, \quad L=l_{1} f\left(z_{1}\right)>0, \quad Z=l_{2} g\left(z_{2}\right)>0, \\
M=\left(q_{1}+h_{1}-\gamma c_{1}\right) \int_{0}^{z_{1}} g\left(z_{2}+\frac{z_{1}-x}{b}\right) f(x) d x>0, \\
R=\left(q_{2}+h_{2}-\gamma c_{2}\right) \int_{0}^{z_{2}} f\left(z_{1}+\frac{z_{2}-x}{a}\right) g(y) d y>0, \\
T=\left(q_{2}+h_{2}-\gamma c_{2}\right) F\left(z_{1}\right) g\left(z_{2}\right)>0 .
\end{gathered}
$$


Then, $\frac{d z_{2}^{1}}{d z_{1}}$ and $\frac{d z_{2}^{2}}{d z_{1}}$ are written as $\frac{d z_{2}^{1}}{d z_{1}}=-\frac{1}{b}-\frac{(K+L)}{M}, \frac{d z_{2}^{2}}{d z_{1}}=\frac{-R}{T+\frac{R}{a}+Z}$. The difference of the derivatives is

$$
\frac{d z_{2}^{2}}{d z_{1}}-\frac{d z_{2}^{1}}{d z_{1}}=\frac{M(T+Z)+b(K+L)\left(T+\frac{R}{a}+Z\right)+\left(\frac{1}{a}-b\right) R M}{b M\left(T+\frac{R}{a}+Z\right)}
$$

which is positive since every term both in the numerator and the denominator are positive. Hence, there exists a unique intersection of the curves $A_{1}\left(z_{1}, z_{2}\right)=0$ and $A_{2}\left(z_{1}, z_{2}\right)=0$ in $\left(z_{1}, z_{2}\right)$ plane.

REMARK 2: Nash equilibrium identified above for the infinite horizon problem is myopic because it is the Nash solution of the static (one-period) game with the following payoff functions of the retailers for every $\left(z_{1}, z_{2}\right)$ pair:

$$
\begin{aligned}
& c_{1} z_{1}+L_{1}\left(z_{1}, z_{2}\right)-\gamma c_{1} \int_{0}^{z_{1}} \int_{0}^{z_{2}+\frac{z_{1}-x}{b}}\left(z_{1}-x-b\left[y-z_{2}\right]^{+}\right) g(y) f(x) d y d x, \\
& c_{2} z_{2}+L_{2}\left(z_{1}, z_{2}\right)-\gamma c_{2} \int_{0}^{z_{2}} \int_{0}^{z_{1}+\frac{z_{2}-y}{a}}\left(z_{2}-y-a\left[x-z_{1}\right]^{+}\right) f(x) g(y) d x d y .
\end{aligned}
$$

The model developed in this section satisfies the conditions presented by Sobel in [13] to guarantee the existence of myopic equilibrium strategies in stochastic games with finite state and action spaces.

Below, an explanation is given for the satisfaction of each condition due to Sobel [13]:

(i) The instantaneous payoff function is the summation of two terms, one term is a function of the actions $\left(z_{1}, z_{2}\right)$ taken and the other is a function of the current state $(I, J)$, namely, $\left(c_{1} z_{1}+L_{1}\left(z_{1}, z_{2}\right)\right)-c_{1} I$ and $\left(c_{2} z_{2}+L_{2}\left(z_{1}, z_{2}\right)\right)-c_{2} J$ for retailers I and II, respectively.

(ii) The transition probabilities do not depend on the current state $(I, J)$ but on the actions $\left(z_{1}, z_{2}\right)$ taken, i.e., for every $n, P\left(\left(I_{n-1}, J_{n-1}\right)=(K, L) \mid\left(I_{n}, J_{n}\right)=\right.$ $\left.(I, J),\left(Q_{1 n}, Q_{2 n}\right)=\left(Q_{1}, Q_{2}\right)\right)$ is equal to

$$
P\left(\left[z_{1}-X-b\left[Y-z_{2}\right]^{+}\right]^{+}=K,\left[z_{2}-Y-a\left[X-z_{1}\right]^{+}\right]^{+}=L\right)
$$

with $z_{1}=I+Q_{1}, z_{2}=J+Q_{2}$.

(iii) From Theorem 1, the static Nash noncooperative game in (7), (8) has an equilibrium.

(iv) Under the equilibrium strategies of the static game, all transitions occur between the states in $\left[0, S_{1}^{*}\right] \times\left[0, S_{2}^{*}\right]$. In other words, equilibrium strategies of the static game are feasible for the states in $\left[0, S_{1}^{*}\right] \times\left[0, S_{2}^{*}\right]$.

A final remark for the substitutable product inventory problem is for comparing the noncooperative case studied up to now in this article with the cooperation case, which is always preferable to the noncooperation case in making the total payoff lower. [But then, the next step in coming up with a solution for this cooperative game would be to find out the way for the retailers to share 
the total payoff amount.] The analysis of this case is simply an extension of the discussion about cooperation in [9] for the multi-period model as shown by the following remark:

REMARK 3: For given order-upto-levels $z_{1 n}=z_{1}$ and $z_{2 n}=z_{2}$ and demand values $X_{n}=x$ and $Y_{n}=y$ for retailers I and II, respectively, in period $n$, the total one-period payoff function in state $\left(I_{n}, J_{n}\right)=(I, J)$ for the cooperative case is

$$
\begin{aligned}
& c_{1}\left(z_{1}-I\right)+c_{2}\left(z_{2}-J\right)+h_{1} I_{n-1}+h_{2} J_{n-1} \\
& \quad+l_{1}\left((1-a)\left[x-z_{1}\right]^{+}+\left[a\left[x-z_{1}\right]^{+}-\left[z_{2}-y\right]^{+}\right]^{+}\right) \\
& \quad+l_{2}\left((1-b)\left[y-z_{2}\right]^{+}+\left[b\left[y-z_{2}\right]^{+}-\left[z_{1}-x\right]^{+}\right]^{+}\right)-q_{1} \min \left\{z_{1}, x+b\left[y-z_{2}\right]^{+}\right\} \\
& \quad-q_{2} \min \left\{z_{2}, y+a\left[x-z_{1}\right]^{+}\right\},
\end{aligned}
$$

where $I_{n-1}=\left[z_{1}-x-b\left[y-z_{2}\right]^{+}\right]^{+}, J_{n-1}=\left[z_{2}-y-a\left[x-z_{1}\right]^{+}\right]^{+}$.

In cases $x \leq z_{1}, y \leq z_{2}$ and $x>z_{1}, y>z_{2}\left(x \leq z_{1}, y>z_{2}\right.$ and $\left.x>z_{1}, y \leq z_{2}\right)$, the total one-period payoff above is equal to (less than or equal to) the summation of the one-period payoff amounts of the two retailers in the strict noncooperative case.

When the retailers cooperate, lost sale cost is not incurred if demand of one product is satisfied by the other product. In case $x \leq z_{1}, y>z_{2},(1-b)\left(y-z_{2}\right)$ is the demand for product 2 lost because customers do not accept substitution and $\left[b\left(y-z_{2}\right)-\left(z_{1}-x\right)\right]^{+}$is the substitutable demand for product 2 which is lost when there is not enough stock of product 1. Similarly, if $x>z_{1}, y \leq z_{2}$, then $(1-a)\left(x-z_{1}\right)$ denotes the amount that can not be substituted by product 2 and $\left(a\left(x-z_{1}\right)-\left(z_{2}-y\right)\right)$ is the substitutable amount which is lost if it is greater than zero.

In order to find an optimal joint strategy of the retailers when they cooperate, one needs to proceed with a single-retailer multi-product model. Finite-horizon dynamic programming formulation would then have the following form:

$$
\begin{aligned}
C_{n}(I, J) & =\min _{\left(z_{1}, z_{2}\right) \geq(I, J)} c_{1}\left(z_{1}-I\right)+c_{2}\left(z_{2}-J\right)+L\left(z_{1}, z_{2}\right) \\
& +\gamma \int_{0}^{\infty} \int_{0}^{\infty} C_{(n-1)}\left(\left[z_{1}-x-b\left[y-z_{2}\right]^{+}\right]^{+},\left[z_{2}-y-a\left[x-z_{1}\right]^{+}\right]^{+}\right) g(y) f(x) d y d x
\end{aligned}
$$

where $C_{n}(I, J)$ is defined as the minimum expected discounted total payoff for the remaining $n$ periods until the end of the horizon and

$$
\begin{aligned}
L\left(z_{1}, z_{2}\right)= & h_{1} E\left(\left[z_{1}-X-b\left[Y-z_{2}\right]^{+}\right]^{+}\right)+h_{2} E\left(\left[z_{2}-Y-a\left[X-z_{1}\right]^{+}\right]^{+}\right) \\
& +l_{1} E\left((1-a)\left[X-z_{1}\right]^{+}+\left[a\left[X-z_{1}\right]^{+}-\left[z_{2}-Y\right]^{+}\right]^{+}\right) \\
& +l_{2} E\left((1-b)\left[Y-z_{2}\right]^{+}+\left[b\left[Y-z_{2}\right]^{+}-\left[z_{1}-X\right]^{+}\right]^{+}\right) \\
& -q_{1} E\left(\min \left\{z_{1}, X+b\left[Y-z_{2}\right]^{+}\right\}\right)-q_{2} E\left(\min \left\{z_{2}, Y+a\left[X-z_{1}\right]^{+}\right\}\right)
\end{aligned}
$$

is the immediate expected payoff except the ordering cost given the order-upto-levels are $z_{1}$ and $z_{2}$. In order to investigate the structure of optimal ordering strategies for this formulation or when $n$ goes to infinity, the analysis should be performed within the context of single-retailer multi-product dynamic inventory control.

\section{CONCLUSION}

In this study, infinite horizon substitutable product inventory problem is formulated as a twoperson nonzero-sum discounted stochastic game and Nash ordering strategies of the retailers are 
investigated within the class of stationary base stock strategies. It is assumed that the setup costs are zero. The analysis is based on minimizing the discounted payoff function of one retailer given that the other retailer is using a stationary base stock strategy. It is shown that his optimal strategy is also a stationary base stock strategy. The existence of a unique Nash equilibrium is proved within the class of stationary base stock strategies. Also, for cooperating retailers, which is as always dominating the noncooperative solution alternatives in the sense of giving lower expected total discounted payoff than the sum of the retailer's payoffs in the noncooperative case, the single retailer inventory control problem is formulated.

The infinite horizon noncooperative model presented in this article is an extension of the singleperiod problem considered in [9]. Parlar conjectured the existence of $(s, S)$-type Nash strategies for multi-period problem in the same article. The work here in this article proves the validity of this conjecture when there is no setup cost. The analysis also shows that the stationary base stock Nash strategies of the retailers are myopic in accordance with the results obtained in [6] for a class of dynamic oligopolies and the generalization of these results in [13].

Relaxation of the constraints under which the substitutable product inventory problem is analyzed in this article underlines future research directions as itemized below:

- The ordering cost is a linear function of the quantity ordered. Analyzing the problem when the setup costs are nonzero and investigating the validity of Parlar's conjecture on the existence of $(s, S)$-type Nash strategies remain as a further research topic.

- The Nash equilibrium identified in this article can be attained if both retailers restrict themselves to stationary base stock strategies. Analysis of the substitutable product inventory problem over a larger strategy space would address the existence of other Nash strategies of different types.

- The discount factor, demand distributions and the substitution probabilities are considered stationary. However, there may be cases where those are nonstationary, e.g., the substitution probabilities might change over time as a function of the actions taken by the retailers. Consideration of the problem under such nonstationary conditions would also lead to the investigation of the problem over larger strategy spaces.

- A natural extension would be the analysis of the problem under the average expected payoff criterion.

- In proceeding along any further research direction, cooperation of the retailers would turn out as an implementable option to be studied as compared to the noncooperative case.

\section{REFERENCES}

[1] R. Bellman, I. Glicksberg, and O. Gross, On the optimal inventory equation, Manage Sci 2 (1955), 83-104.

[2] J.A. Filar, T.A. Schultz, F. Thuijsman, and D.J. Vrieze, Nonlinear programming and stationary equilibria in stochastic games, Math Program 50 (1991), 227-238.

[3] D.L. Iglehart, The optimality of $(s, S)$ policies in the infinite horizon dynamic inventory problem, Manage Sci 9 (1963), 259-267.

[4] E. Ignall and A.F. Veinott, Optimality of myopic inventory policies for several substitute products, Manage Sci 15 (1969), 284-304.

[5] M. Khouja, A. Mehrez, and G. Rabinowitz, A two-item newsboy problem with substitutability, Int J Prod Econ 44 (1996), 267-275. 
[6] A.P. Kirman and M.J. Sobel, Dynamic oligopoly with inventories, Econometrica 42 (1974), 279-287.

[7] A.R. McGillivray and E.A. Silver, Some concepts for inventory control under substitutable demand, Infor 16 (1978), 47-63.

[8] M. Parlar, Optimal ordering policies for a perishable and substitutable product: A Markov decision model, Infor 23 (1985), 182-195.

[9] M. Parlar, Game theoretic analysis of the substitutable product inventory problem with random demands, Nav Res Logistics 35 (1988), 397-409.

[10] M. Parlar and S.K. Goyal, Optimal ordering decisions for two substitutable products with stochastic demands, Opsearch 21 (1984), 1-15.

[11] T.E.S. Raghavan and J.A. Filar, Algorithms for stochastic games - a survey, ZOR-Methods Models Oper Res 35 (1991), 437-472.

[12] H. Scarf, The optimality of $(s, S)$ policies in the dynamic inventory problem, Math Methods Soc Sci (1960), 196-202.

[13] M.J. Sobel, Myopic solutions of Markov decision processes and stochastic games, Oper Res 29 (1981), 995-1009.

[14] A.F. Veinott, Optimality policy for multi-product, dynamic, nonstationary inventory problem, Manage Sci 12 (1965), 206-222.

[15] Q. Wang and M. Parlar, A three-person game theory model arising in stochastic inventory control theory, Eur J Oper Res 76 (1994), 83-97. 